\title{
Situation Awareness and Secondary Task Performance While Driving
}

\author{
Martin R.K. Baumann, Diana Rösler, and Josef F. Krems \\ Chemnitz University of Technology, Institute of Psychology, \\ D-09107 Chemnitz, Germany \\ \{Krems, Diana.Roesler, Martin.Baumann\} @phil.tu-chemnitz.de
}

\begin{abstract}
For safe driving it is necessary that the drivers perceive the relevant objects of a situation, comprehend the meaning of these objects to form a holistic understanding of the current situation, and predict the future development of the situation. A concept that aims to describe and integrate these different cognitive processes is situation awareness, for example [1]. According to this concept it is assumed that a mental representation is constructed, maintained, and updated while driving. Attentional and working memory (WM) resources are involved in these processes. If secondary tasks performed while driving impose significant load on visual attention and WM, then one can expect that situation awareness is impaired. We investigated these predictions in 2 experiments. The results show that both cognitively and visually demanding tasks interfere with the maintenance of a correct situation model in memory. Visually demanding tasks do not always seem to interfere with visual attention processes in ways that lead to degraded situation awareness.
\end{abstract}

Keywords: Visual demand, situation awareness, secondary tasks, driving.

\section{Introduction}

For safe driving it is necessary that drivers perceive, identify, and correctly interpret the relevant objects and elements of the current traffic situation and that they construct expectations about the future development of the current situation to adapt their own driving behavior to the situation. Such elements may be other traffic participants, the surface of the street, or traffic signs. Situation awareness has been recently proposed in aviation psychology as a concept that aims to describe and integrate these different cognitive processes within a common framework ([1], [2], [3]).

According to Endsley three functions of situation awareness can be distinguished [1]. The first function involves the perception of the status, the attributes, and the dynamics of the relevant situation elements. The second function, comprehension, aims to integrate the different situation elements into a holistic picture of the situation, resulting in the comprehension of the meaning of the different elements. The third function of situation awareness is to construct expectations about the future behavior of the situation elements on the basis of the comprehension of the situation. 
Whereas Endsley [1] describes different cognitive resources and mechanisms that might be involved in constructing and maintaining situation awareness her model is rather vague about the nature of these processes and how the different functions of situation awareness are realized. Baumann and Krems [4] proposed on the basis of Kintsch's theory of discourse comprehension [5] a model of situation awareness that assumes that situation awareness is constructed by a comprehension process (cf. [2]). Perceived information activates knowledge stored in long-term memory (LTM) that is linked to the perceived information. From this activated knowledge network a coherent representation is constructed by a constraint satisfaction process. This process constrains the spreading of activation leading to the activation of compatible and to the suppression of incompatible knowledge elements of the activated knowledge network. The result is a coherent mental representation of the current situation, the situation model. For example, an event such as a traffic light turning yellow might activate at first two incompatible interpretations, "I have to decelerate to stop before the traffic light" and "I have to accelerate to pass the crossroads before the traffic light turns red". These two interpretations might receive additional activation from other knowledge elements. For example, if the driver knows that the police monitor this crossroads, that knowledge will additionally activate the deceleration interpretation and at the same time inhibit the acceleration interpretation. With this additional activation the deceleration interpretation "wins" and the acceleration interpretation will be inhibited. The experienced driver's knowledge presumably also includes expectations about the future development of situations that are linked to certain types of situation and activated when these types of situations, such as approaching a traffic light, are encountered. In this sense the same process that serves the comprehension function of situation awareness also serves the prediction function, especially in routine driving situations for which the driver already acquired relevant knowledge. The situation model is the basis for the driver's actions. These actions and the dynamics of the driving task make it necessary to update the situation model constantly.

This comprehension based view of situation awareness emphasizes the importance of cognitive resources for the construction and maintenance of situation awareness besides visual attention. The integration of new information into the situation model, the updating of the model, and the use of the situation model as a basis for action selection all need working memory (WM) resources. Visual perception and attention processes serve the perception function of situation awareness. Both the driver's visual and cognitive resources need to be available to construct a proper situation model that is necessary for driver's safety. But in modern cars more and more driver support and information systems are available to the driver confronting the driver with more and more in-car tasks. Performing these in-car tasks while driving creates a dual task situation for the driver that could lead to the impairment of situation awareness if the task strongly involves visual attention or cognitive resources. An incar task that is highly visually demanding may lead to a degraded situation model as many relevant elements of the traffic situation will simply not be perceived and therefore not integrated into the situation model. A highly cognitively demanding incar task should interfere with the activation of knowledge, the maintenance of the situation model in WM, and the updating of the model. In the design process of such in-car tasks it is therefore important to consider the effects of such tasks on situation 
awareness to avoid too visually and / or cognitively demanding tasks. The first goal of this paper is to describe a procedure that should allow to evaluate the visual and cognitive demands associated with such tasks. A sample of tasks was evaluated with this procedure. Then this sample of tasks had to be performed while driving and the effects of these tasks in driver's situation awareness was assessed. The second goal of this paper is accordingly to test the predictions of the comprehension based situation awareness model [4] about the effects of visually and cognitively demanding tasks on situation awareness.

The evaluation procedure uses a dual-task technique to measure the visual and cognitive demands of in-car tasks. The basic idea is to measure the degree of interference between the processes involved in the construction and maintenance of situation awareness and those that are involved in performing in-car tasks. Participants have to perform the in-car tasks in the laboratory as primary tasks. The secondary task is used to measure both the residual visual attention and WM resources not utilized by the primary in-car task. We used a 1-back task as secondary task. In this task participants are presented with one of four different visual stimuli on each trial. The participants' task is to indicate whether the current stimulus is identical to the one presented in the previous trial. This task seemed suitable for assessing situation awareness relevant interference effects of in-car tasks for the following reasons: The reaction to a stimulus is context dependent, that is a minimum situation model has to be kept in memory and this situation model has to be updated frequently. Thus, the task involves two basic components of situation awareness processes.

The 1-back task yields two measures that are supposed to allow assessment of the visual and cognitive demands of the primary task. First, one can look at the proportion of visual stimuli responded to, not differentiating whether the response was correct or not. This detection rate is used as a measure of the visual demand of the in-car task. If the in-car task is highly visually demanding the participant will simply miss many of the visual stimuli. In this regard, the task is similar to a laboratory variant of the peripheral detection task (PDT, [6]) that was previously developed to measure only visual demand of in-car tasks. The proportion of correct responses of all shown responses, the hit rate, is used as a measure of the cognitive demands of the in-car task. If the task is highly demanding the participant may often forget the previous stimulus or may frequently fail to update the stimulus. This should lead to more false responses to the visual stimuli.

\section{Experimental Studies}

We conducted two experiments. In the first experiment three tasks differing in visual and cognitive demand were evaluated with the procedure described above. In the second experiment these tasks were performed while driving in a driving simulator and different measures of situation awareness were collected. The aim of these two experiments was to test the prediction that tasks that were evaluated as highly visually demanding should show an impairment of the perception function of situation awareness whereas tasks that were evaluated as highly cognitive demanding should show an impairment in maintaining and updating the situation model. 


\subsection{Experiment 1}

The aim of Experiment 1 was to evaluate the visual and cognitive demands of different tasks using a 1-back task as the measurement procedure, where the participants had to compare the current stimulus with the one that was presented immediately before.

Method. In this experiment 21 participants performed three in-car tasks together with a 1-back task. The participants' age ranged from 19 to 30 years. There were 11 women and 10 men.

The in-car tasks were Listening to a spoken text, asking an automated train information system with automated Speech recognition for the arrival time of a train, and writing a short text with a PDA using its touch screen and stylus.

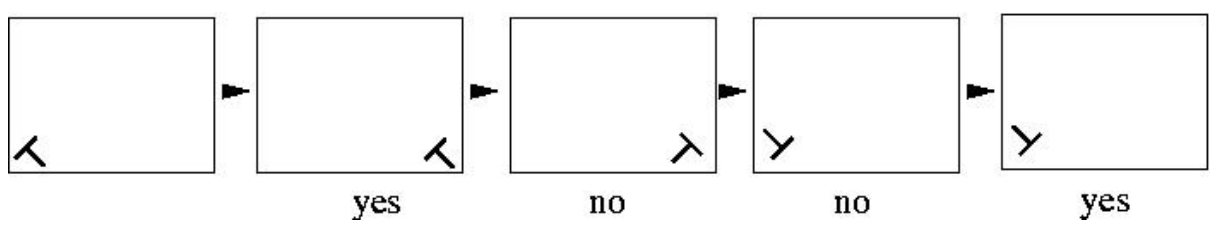

Fig. 1. Example of a 1-back task stimulus sequence with the correct answer for each stimulus presentation

In the 1-back task the participants were presented with T-like visual stimuli that were rotated by $45^{\circ}, 135^{\circ}, 225^{\circ}$, or $315^{\circ}$ (see Fig. 1). The size of the stimuli was $1.2^{\circ}$ of visual angle. Each stimulus was presented for $500 \mathrm{msec}$. The interval between two stimulus presentations varied randomly between 1 and $2 \mathrm{sec}$. They were presented at either $23^{\circ}$ of visual angle to the left or to the right of the participant at a distance of $2.5 \mathrm{~m}$. The participants' task was to decide for each new stimulus whether it was identical to the one presented in the trial before. The probability for each new stimulus being identical to the one presented before was .5. In this task each stimulus acts as the context for the next stimulus and the context could change after each stimulus presentation. Therefore, for each stimulus presentation participants had to decide whether this stimulus was identical to the previous one and then they had to encode the new stimulus as the new context, get rid of the old context, keep the current context in WM until the next stimulus was presented, and then encode this stimulus as the new context. As explained above the detection rate was used as a measure of the visual demand of the respective in-car task and the hit rate was used as a measure of the cognitive demand of the in-car task.

Table 1. Detection and hit rate in the 1-back task for the different in-car tasks

\begin{tabular}{lll}
\hline & Detection rate & Hit rate \\
\hline Baseline & .84 & .89 \\
Listening & .78 & .84 \\
Speech interaction & .68 & .77 \\
PDA writing & .17 & .47 \\
\hline
\end{tabular}


Results and Discussion. The detection and the hit rates for the different in-car tasks are shown in Table 1 . The detection rates of the different tasks differed significantly, $\mathrm{F}(2.25,42.76)=121.67, \mathrm{p}<0.001$. All tasks were significantly different from each other, with one exception: baseline and listening did not differ significantly. As expected the task that involved the interaction with a visual display, the PDA writing task, had the lowest detection rate. Additionally, the difference between the listening and the speech interaction task was also significant. As there is no difference in the visual demand of both tasks this difference can only be attributed to the difference in cognitive demand between tasks. Therefore, this difference indicates that the detection rate is to some extent also sensitive to the cognitive demands of tasks.

Only the hit rate data from 17 participants were analyzed as four participants showed no reaction to the 1-back task stimuli during the PDA writing task. As for the detection rate the tasks showed an overall difference, $F(1.37,21.93)=21.23, p<$ 0.001 . There was no significant difference between baseline and listening. All other tasks had a significantly lower hit rate than the baseline. Additionally, the listening and the speech task significantly differed from the PDA writing task.

The results indicate that detection rate might be a valid measure of the visual demand of tasks, although it is also influenced by the cognitive demand of the tasks. The interpretation of the hit rate as a measure of the cognitive demand of tasks is much more difficult. First, the task that was associated with a low detection rate (PDA writing) had also a low hit rate. Second, there was no significant difference between the listening and the speech interaction task despite the fact that the cognitive demands of both tasks clearly differ and that there was a significant difference between both tasks in the detection rate that was attributed to the different cognitive demands of both tasks. It might be the case that the hit rate of the 1-back task was not sensitive enough to detect the difference in cognitive demand between the listening and the speech task.

\subsection{Experiment 2}

Experiment 2 was a driving simulator study. The aims of this experiment were to examine the effects of the in-car tasks of Experiment 1 on situation awareness and to compare these effects with the effects these tasks had on the detection and the hit rate of the 1-back task in Experiment 1. The driving scenario consisted of driving in the middle lane of a motorway with three lanes in each direction. The participants were instructed to drive in the middle lane at $110 \mathrm{~km} / \mathrm{h}$ whenever possible. Situation awareness was measured by repeatedly interrupting the driving simulation and asking the driver questions about the number of cars in different lanes. There were four relevant locations that were tested. The four locations were: i) left lane behind the driver, ii) left lane in front of the driver, iii) middle lane in front of the driver, and iv) right lane in front of the driver. After each interruption only one of the four possible locations was tested.

The response accuracy for the locations in front of the driver should indicate how the perception function of situation awareness is influenced by an in-car task. This information is always visible and incorrect answers should mainly arise from failures in perception and visual attention. The response accuracy for the location behind the driver should indicate the effects of the in-car tasks on the maintenance and updating 
of the situation model in WM. This information has to be kept in memory, presumably in working memory, until the driver updates the information by looking into the interior or the left external mirror. Incorrect answers should arise from the driver either not looking into the mirrors or forgetting what was encoded before. Therefore, we expected first that the visually demanding PDA writing task should lead to more incorrect answers to questions regarding the front locations than the not visually demanding tasks listening and speech interaction. Second, we expected that both visually and cognitively demanding tasks, both the PDA writing and the speech interaction task, should lead to more incorrect answers to questions regarding the rear location than the less cognitively demanding task listening.

Method. 19 participants performed the same three in-car tasks that were used in Experiment 1 while driving on a motorway in a driving simulator. 15 of the participants were female. The mean age was $23.79(\mathrm{SD}=2.82)$.

For the driving simulation we used the driving simulator of Systems Technologies Inc. The simulation was presented on three 19"-TFT screens providing a projection of $135^{\circ}$ of visual angle.

Participants performed twelve trials of each in-car task. For each trial a new ride was started. During each ride some critical events happened, such as braking of the lead car, to make sure that the participants paid attention to the driving task. After $2000 \mathrm{~m}, 3000 \mathrm{~m}$, or $4000 \mathrm{~m}$ of driving the simulation was suddenly interrupted and the participants were asked about the number of cars on one of the four positions mentioned above. Each position was tested three times during each in-car task block.

Results and Discussion. A 4 (locations) x 4 (in-car task blocks) repeated measures ANOVA was performed to analyze the data. There was a clear effect of location, $\mathrm{F}(3$, $54)=10.68, \mathrm{p}<0.001$. The frequency of correct answers was largest for the middle lane and lowest for the left lane behind the driver. The in-car tasks did not differ significantly. But there was a significant interaction between location and in-car task, $\mathrm{F}(9,162)=2.26, \mathrm{p}=0.02$. This was due to the differences between tasks that were much greater at the locations behind and right in front of the driver than for the locations in the middle and left in front of the driver. The results for these two positions are shown in Table 2. Inspecting the results for the rear left position, one can see that in agreement with the results of Experiment 1 the accuracies for the speech interaction and the PDA writing task were reduced compared to the baseline condition. Accuracy was not reduced for the listening task as was expected from the results in Experiment 1. The results for the front right position in Experiment 1 predicted that the accuracy for the PDA writing task should be reduced because of its visual demand. But this was not the case.

Table 2. Accuracy of answers to the questions about the number of cars in the two lanes for the different in-car tasks

\begin{tabular}{lll}
\hline & Right front lane & Rear left lane \\
\hline Baseline & .75 & .72 \\
Listening & .70 & .74 \\
Speech interaction & .62 & .55 \\
PDA writing & .75 & .58 \\
\hline
\end{tabular}




\section{Summary and Conclusions}

Two experiments were described that tested predictions about the effects of in-car tasks on situation awareness. These predictions were derived from a comprehension based model of situation awareness [4]. In the first experiment an experimental paradigm was tested and used to evaluate the visual and cognitive demands of potential in-car tasks. This paradigm is based on the use of a 1-back task that has to be performed concurrently with an in-car task to measure the visual and cognitive demands of this in-car task. We argued that the detection rate for the 1-back task stimuli depends mainly on the visual demand of the concurrently performed in-car task. The hit rate, that is the proportion of correct responses of all responses made, should be sensitive to the cognitive demand of the in-car task. Consistent with our expectations we found a decreased detection rate for the visually demanding task and a decreased hit rate for the cognitively demanding tasks.

In the second experiment the effect of these tasks on drivers' situation awareness was evaluated in a driving simulator study. Participants had to perform the same tasks that were used in Experiment 1 while driving in a driving simulator. Situation awareness was measured by interrupting the simulation and asking participants about the number of cars on the surrounding lanes.

Contrary to our expectations we found that the PDA writing task that showed a significant decrement in the detection rate in the first experiment led to no decrement in accuracy when the driver was asked for the number of cars in front - irrespective of the lane. It might be that participants were able to use peripheral vision and short glances to watch the traffic situation in front of them while performing the PDA writing task (cf. [7], [8]). In agreement with the model predictions we found that those tasks that were evaluated as highly visually or cognitively demanding, the PDA writing resp. the speech interaction task, lead to more errors in recalling the number of cars at the rear position. The visual demand of the PDA writing task might have reduced the number of glances to the mirrors leading to a reduced integration of information about the rear situation into the situation model. The cognitive load of the speech interaction task presumably led to an increased forgetting of encoded information. Another possibility could be that the cognitive demand of the task led drivers to reduce watching the mirrors and concentrate their glances on a region straight ahead. Therefore, information about the rear situation was gathered to a lesser extent. Such a type of reaction to high cognitive load while driving has been found in other studies on the effect of cognitive demand on driving behavior (e.g, [9]).

The results of the two experiments demonstrate that a task such as the n-back task might be suitable as a measure of the cognitive and the visual demands of tasks. But they also demonstrate that the effects of visual and cognitive task demands on measures of situation awareness while driving are complex and sometimes counterintuitive. These effects have to be examined in more detail using pseudo-incar tasks with a better controlled profile of visual and cognitive demands. 


\section{References}

1. Endsley, M.R.: Towards a theory of situation awareness in dynamic systems. Hum Factors 37, 32-64 (1995)

2. Adams, M.J., Tenney, Y.J., Pew, R.W.: Situation awareness and the cognitive management of complex systems. Hum Factors 37, 85-104 (1995)

3. Bailly, B., Bellet, T., Goupil, C.: Driver's mental representations: Experimental study and training perspectives. In: Dorn, L. (ed.): Driver behavior and training. Ashgate, Aldershot (2003)

4. Baumann, M., Krems, J.F.: Situation awareness and driving: A cognitive model. In: Cacciabue, C., Re, C. (eds.): Critical issues in advanced automotive systems and humancentred design. Springer, London (in press)

5. Kintsch, W.: Comprehension: A paradigm for cognition. Cambridge University Press, New York (1998)

6. Baumann, M., Rösler, D., Jahn, G., Krems, J.F.: Assessing driver distraction using occlusion method and peripheral detection task. In: Strasser, H., Kluth, K., Rausch, H., Bubb, H. (eds.) Quality of work and products in enterprises of the future, pp. 53-56. Ergonomia Verlag, Stuttgart (2003)

7. Chiang, D.P., Brooks, A.M., Weir, D.H.: On the highway measures of driver glance behaviour with an example automobile navigation system. Appl. Ergon. 35, 215-224 (2004)

8. Wierwille, W.: Visual and manual demands of in-car controls and displays. In: Peacock, B., Karwowski, W. (eds.): Automotive ergonomics, Taylor and Francis, London (1993)

9. Victor, T.W., Harbluk, J.L., Engström, J.A.: Sensitivity of eye-movement measures to invehicle task difficulty. Transport Res. F 8, 167-190 (2005) 